\title{
Sommerflor 2019 im Eingangsbereich und am Tropicarium
}

\author{
Hilke Steinecke
}

\section{Abstract}

During the summer months, the Palmengarten displays various beds of summer flowers. The plants are grown in the nursery of the Palmengarten. The colour concept varies from year to year. In summer 2019, especially Leonotis nepetifolia and Salvia uliginosa were especially showy plants in the summer beds.

\section{Zusammenfassung}

Im Sommerhalbjahr zeigt der Palmengarten auf verschiedenen Beeten einen Sommerflor. Die Pflanzen hierfür werden in der Gärtnerei des Palmengartens herangezogen. Das Farbkonzept ist jedes Jahr etwas anders. Im Sommer 2019 waren vor allem Leonotis nepetifolius und Salvia uliginosa zwei recht hohe auffällige und üppig gedeihende Gewächse im Sommerflor.

\section{Blütenpracht aus der Palmengarten-Gärtnerei}

Jedes Jahr neu zeigt der Palmengarten von Mitte Mai bis etwa Anfang Oktober auf verschiedenen Beeten einen üppigen Sommerflor. Beete wie das Blumenparterre vor dem Gesellschaftshaus, das von unseren Auszubildenden geplante Azubibeet zwischen Café Siesmayer und Palmenhaus, die kleinen Beete rund um das Eingangsbecken und um die Tanzenden Wasser sowie die Beete am Tropicarium werden mit diversen Zierpflanzen bestückt, die in der Gärtnerei des Palmengartens extra für diesen Zweck herangezogen werden. Dabei kommen insgesamt mehrere Zehntausend Pflänzchen zusammen.

Immer wieder gibt es Überraschungen im Sommerflor zu entdecken. Zusammen mit Indischem

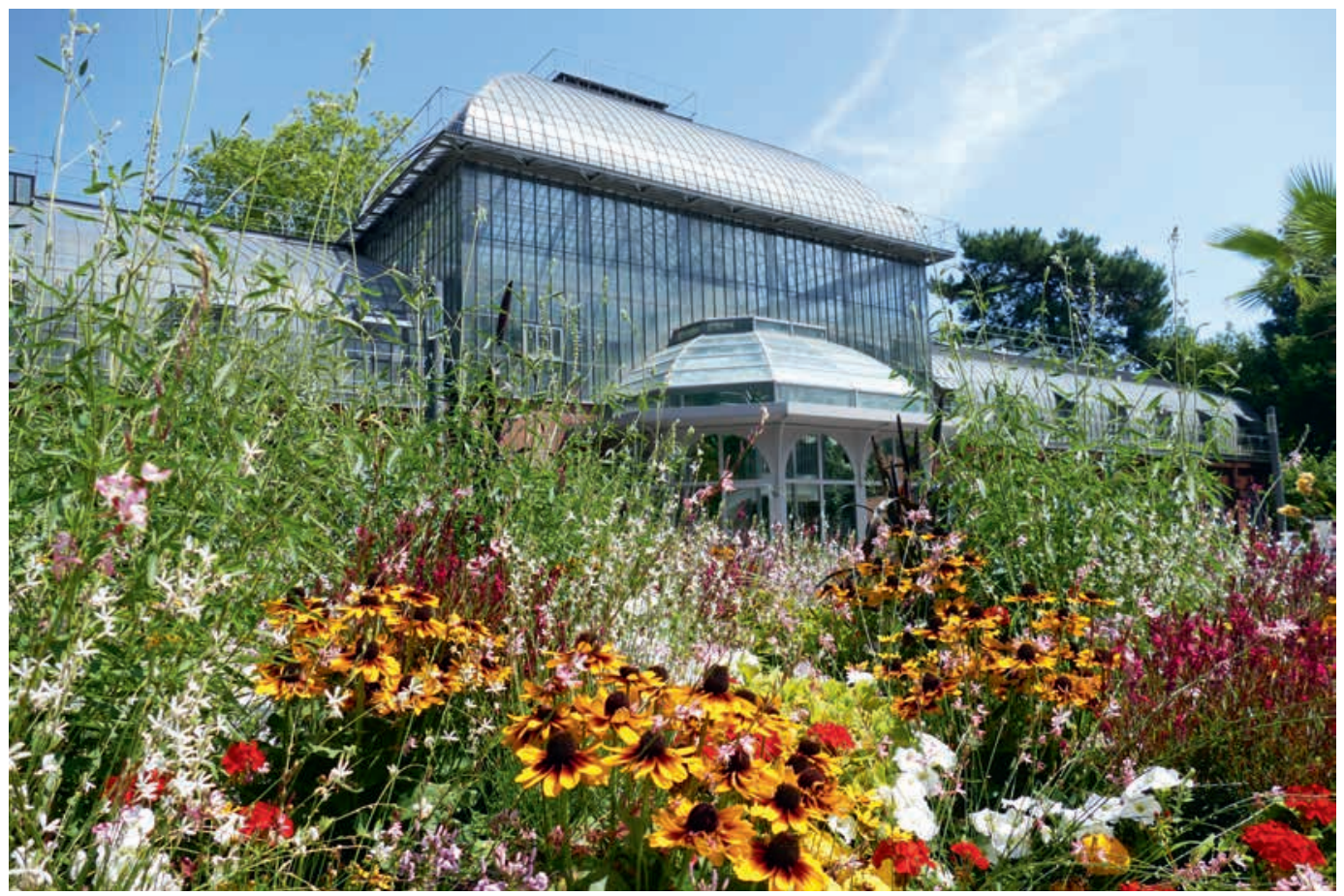

Abb. 1: Im Sommer blüht es am Eingang Siesmayerstraße sehr üppig, besonders auffällig sind Rudbeckien in Gelb- und Orange-Tönen. (Foto: H. STEINEcke) 


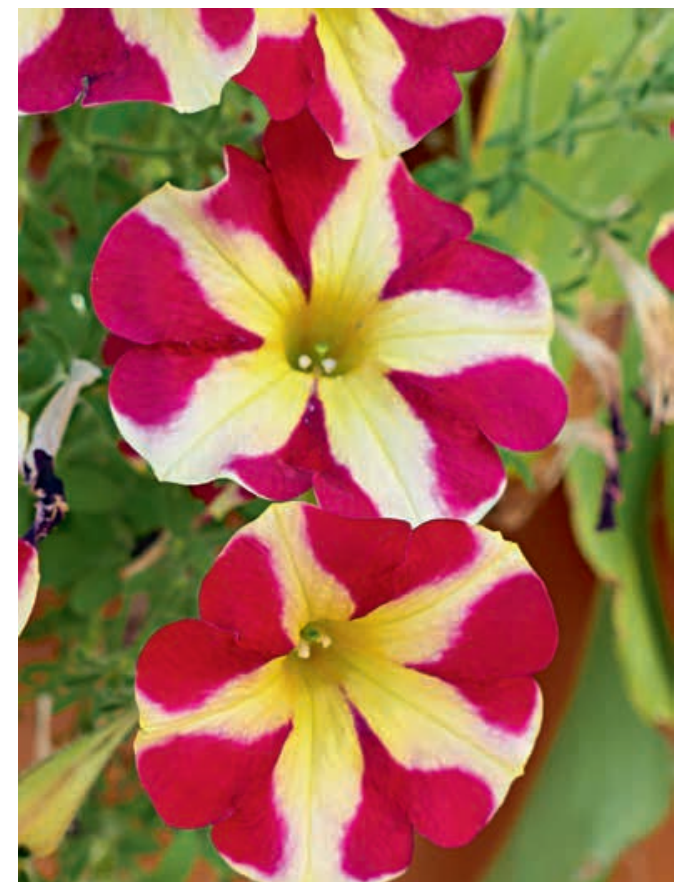

Abb. 2: Petunia 'Pegasus Queen of Hearts' mit fünf roten Herzen auf der Blüte. (Foto: H. Steinecke)

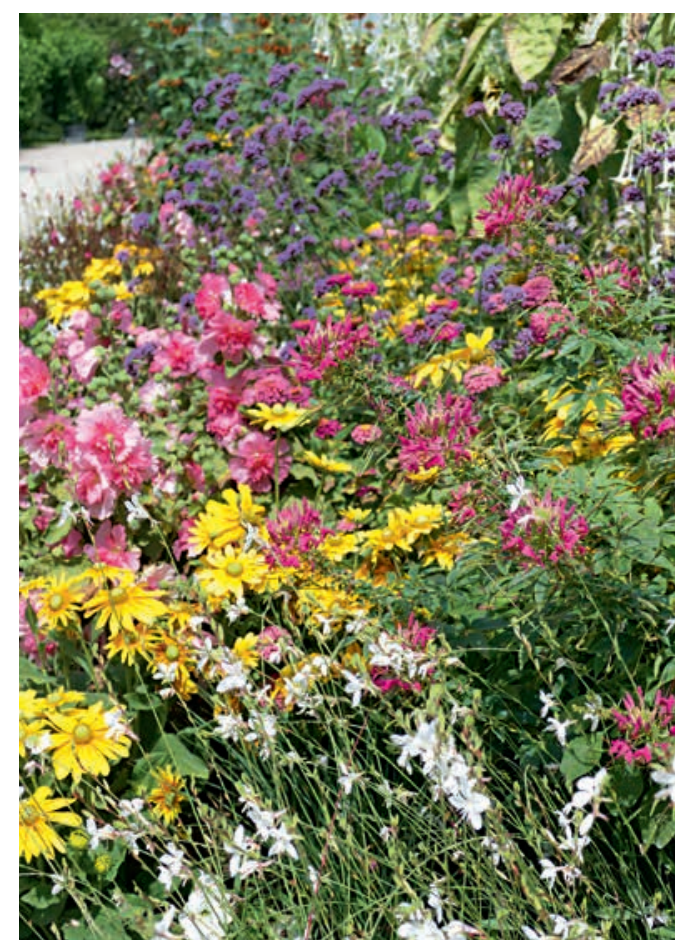

Abb. 3: Ausschnitt aus der Bepflanzung längs des Tropicariums. (Foto: H. Steinecke)
Blumenrohr im Kübel begrüßte eine besondere Petunie die Besucherinnen und Besucher in den Ecken des Eingangsbrunnens. Bei der Frage, welches Muster man in der Petunienblüte erkennen würde, kam meist die Antwort „gelber Stern oder Steuerrad auf rotem Grund“. Einmal darauf aufmerksam gemacht, erkennt man in der Blüte aber einen perfekten Kranz aus fünf roten Herzen auf hellgelbem Untergrund. Die Sorte heißt deshalb auch Petunia 'Pegasus Queen of Hearts'.

Schon seit vielen Jahren hat sich im Eingangsbereich und am Tropicarium eine Millefleurs-Bepflanzung bewährt. Dabei wird jedes Jahr ein etwas unterschiedliches Farbkonzept mit wechselnden Arten und Sorten umgesetzt.

\section{Millefleurs-Pflanzungen}

Farbenfrohe Millefleurs-Pflanzungen wirken durch ihre Leichtigkeit. Sie haben ihren Ursprung als sogenannte Emaille-Pflanzung in der späten Barockzeit sowie im Rokkoko. In diese Pflanzungen wurden früher häufig neue und besonders kostbare Pflanzen integriert. Von vielen Neuheiten war oft nur wenig über Habitus und Blütezeit bekannt, so dass sich im Laufe des Jahres in der Pflanzung gelegentlich ein Durcheinander entwickelte, das aber auch seinen besonderen Reiz hatte.

Im 20. Jahrhundert gerieten die manchmal etwas unordentlich wirkenden Millefleurs-Pflanzungen außer Mode. Erst seit der Internationalen Gartenbauausstellung in München 1983 wurden Millefleurs-Pflanzungen in Deutschland wieder deutlich beliebter. Neben der farblichen Abstimmung kennzeichnet eine solche Pflanzung die sorgfältige Höhenstaffelung zwischen etwa 20 und $80 \mathrm{~cm}$, wobei die höher wachsenden Pflanzen in lockeren kleinen Gruppen zusammenstehen sollten. So wirkt das Ensemble wie ein riesiger bunter Blumenstrauß. Farbe und Form der Blüten sollten sich von der Waagerechten zur Senkrechten vom Schweren (dunkle Farben) zum Leichten (helle Farben) entwickeln. Sehr gut geeignet als halbhoher Partner zu den niedrigen Teppichpflanzen sind Cosmos bipinnatus oder Tabak (z. B. Nicotiana sylvestris), beide sind regelmäßig in unseren Beeten zu sehen. Die Kunst einer 
guten Millefleurs-Pflanzung besteht darin, dass sie mit einem gewissen jahreszeitlichen Wechsel, während der gesamten Sommersaison, blüht. Sehr beruhigend zwischen den bunten Blüten wirken verschiedenen Blattpflanze, darunter Greiskraut (Senecio) mit silbergrauen Blättern, Zier-Süßkartoffeln (Ipomoea batatas) oder Gräser (z. B. Pennisetum glaucum). Für die Randbereiche eignen sich Kontrastfarben und für die Beetmitte eine einheitliche Farbgebung. Es wirkt sehr gut, wenn sich im Zentrum des Beetes mittelhohe und hohe Blumen in der Kontrastfarbe der Kanten entwickeln können. In diesen herausragenden „Sträußen“ sollten bei entsprechendem Platzangebot nicht Einzelpflanzen, sondern mehrere Pflanzen einer Sorte zusammenstehen. Innerhalb der Pflanzung können sich straußartige Gruppen wiederholen.

Das Interessante an unserer Pflanzung ist, dass im Laufe der Vegetationszeit immer wieder verschiedene Gewächse besonders auffällig sind und das Bild prägen und es niemals langweilig wird. Kurz nach der Pflanzung im Mai sind naturgemäß alle Pflanzen noch recht niedrig, ab dem Sommer ragen dann immer mehr höhere Individuen heraus. In diesem Sommer waren es vor allem verschiedene Sonnenblumen (Helianthus annuus), die allerdings wegen der hohen Sommertemperaturen recht schnell verblüht waren und im Juni bereits fruchteten. Die Kerne waren bei Vögeln als Futter sehr beliebt. Im Spätsommer setzten sich zwischen den gelborangen Blütenkörbchen der Sonnenhüte (Rudbeckia hirta) verschiedenfarbiger Zinnien (Zinnia elegans), weiß- bzw. rosablütige Steppenkerzen (Gaura lindheimeri) und besonders die hohen Blütenstände des Ziertabaks (Nicotiana sylvestris) durch.

Einige bewährte Sorten im Sommerflor 2019 waren Chamaesyce hypericifolia 'Montblanc', 'Snow Mountain', Coreopsis grandiflora 'Sunkiss', Echinacea purpurea 'Feeling Pink', Gaura lindheimeri 'Gambit Rose', 'Gambit White', Ipomoea batatas 'Emerald Lace', Liatris spicata 'Kobold', Lobelia erinus 'Laura Purple', Rudbeckia x hybrida 'Summerdaysies Red Bicolor', Tagetes erecta 'Antigua Yellow', Verbena bonariensis 'Violetta', Zinnia elegans 'Oklahoma White', 'Oklahoma Pink'.

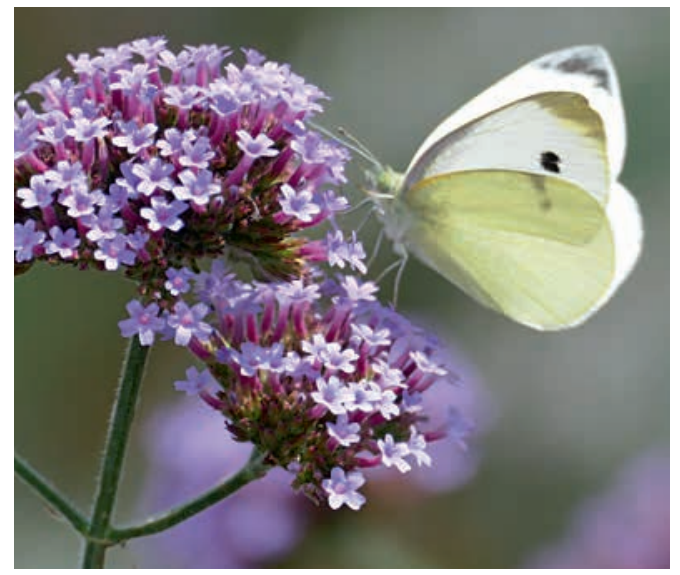

Abb. 4: Verbena bonariensis 'Violetta' trägt sehr gut zur Höhenstaffelung des Sommerblumenbeetes bei und ist gleichzeitig bei Schmetterlingen wie diesem Kohlweißling als Nektarpflanze beliebt. (Foto: H. Steinecke)

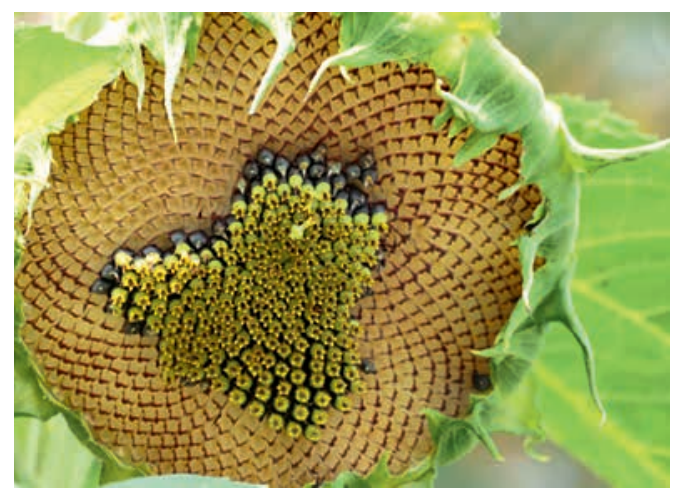

Abb. 5: Sonnenblumenkerne sind bei Vögeln als Futter sehr beliebt, manchmal bleibt nur noch ein Herz stehen. (Foto: H. STEINECKe)

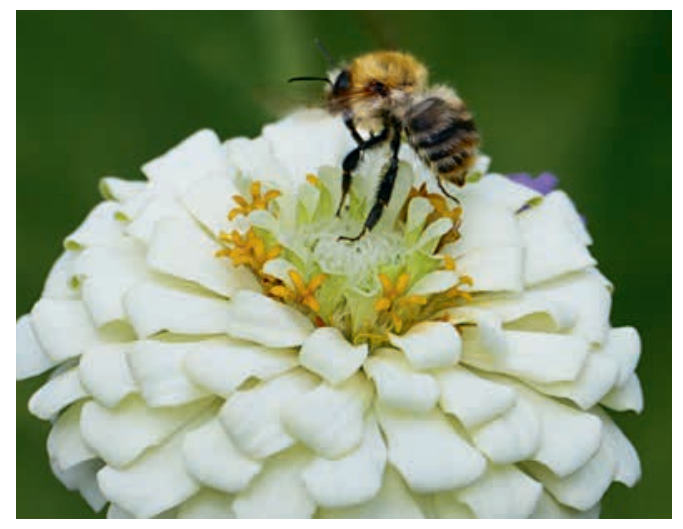

Abb. 6: Zinnia elegans 'Oklahoma White'.

(Foto: H. Steinecke) 


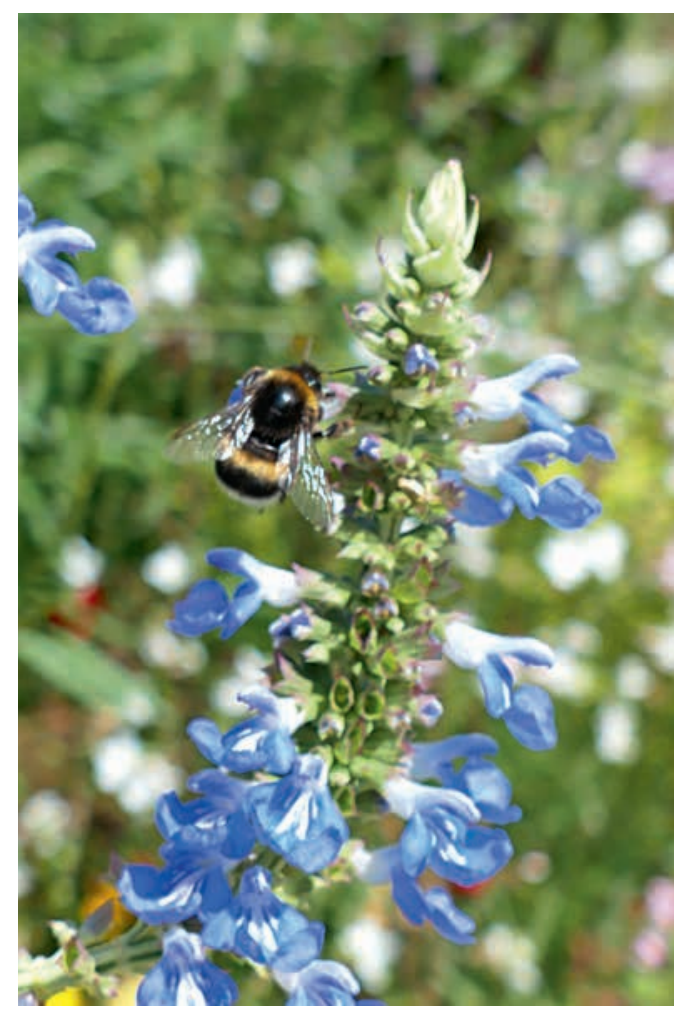

Abb. 7: Erdhummel im Anflug auf den HummelschaukelSalbei (Salvia uliginosa). (Foto. H. STEInecke)

\section{Hummelschaukel (Salvia uliginosa)}

In der Mitte der Beete rund um den Eingangsbrunnen wucherte gegen Ende der Saison eine blaue Blume, die besonders bei Hummeln als Nektarpflanze beliebt ist. Es handelt sich um die Hummelschaukel, die ihren Namen deshalb erhalten hat, weil dicke schwere Hummeln, die sich auf den Blüten niedergelassen haben, den Blütenstand zum Schwingen bringen können. Der in Südamerika heimische Hummelschaukel-Salbei wächst bevorzugt auf sonnigen, feuchten Wiesen. Er gedeiht aber auch auf normalen, nährstoffreichen, nicht zu trockenen Böden und ist bei uns als Kübelpflanze geeignet. Gern wird er aber auch einjährig in Sommerblumen-Beeten kultiviert. Die Pflanzen können im Gewächshaus überwintert werden. Sie sind nur bis etwa $-10^{\circ} \mathrm{C}$ winterhart. Die himmelblauen Blüten sind essbar. Wegen ihrer außergewöhnlichen Farbe sind sie eine interessante Dekoration für Salate. Auch die Blätter haben

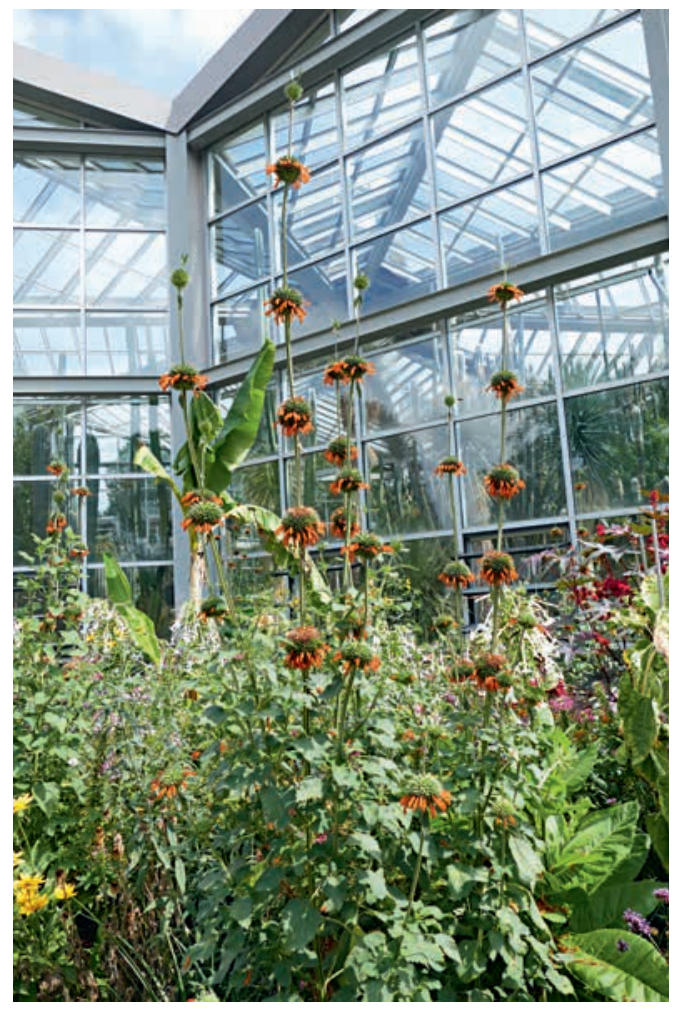

Abb. 8: Die hohen Blütenstände des Löwenohrs (Leonotis nepetifolia). (Foto. H. STEINECKe)

einen pfeffrig-scharfen Geschmack. Deshalb wird die Art auch Pfeffer-Salbei genannt.

Pfeffer-Salbei blüht vom Hochsommer bis zum Oktober und erreicht dann Höhen von 1,5-2 m. Gewöhnlich wächst dieser Salbei zu dichten, buschigen Stauden heran. Außer im Sommerflor ist Salvia uliginosa auch im Steingarten in der Nähe des Mammutblattes (Gunnera) anzutreffen. $\mathrm{Ob}$ sie, hier ausgepflanzt und mit entsprechendem Winterschutz versehen, den kommenden Winter übersteht, ist natürlich von der Härte des nächsten Winters abhängig.

\section{Löwenohr (Leonotis nepetifolia), herausragend schön}

Erst relativ spät im Jahr zeigten sich die etwa $2 \mathrm{~m}$ hohen Blütenstände des Löwenohrs mit ihren auffällig leuchtend orangen, schmalen Blüten. Die Art wurde früher bei uns regelmäßig im SommerSukkulenten-Garten als Unterpflanzung gezeigt, 


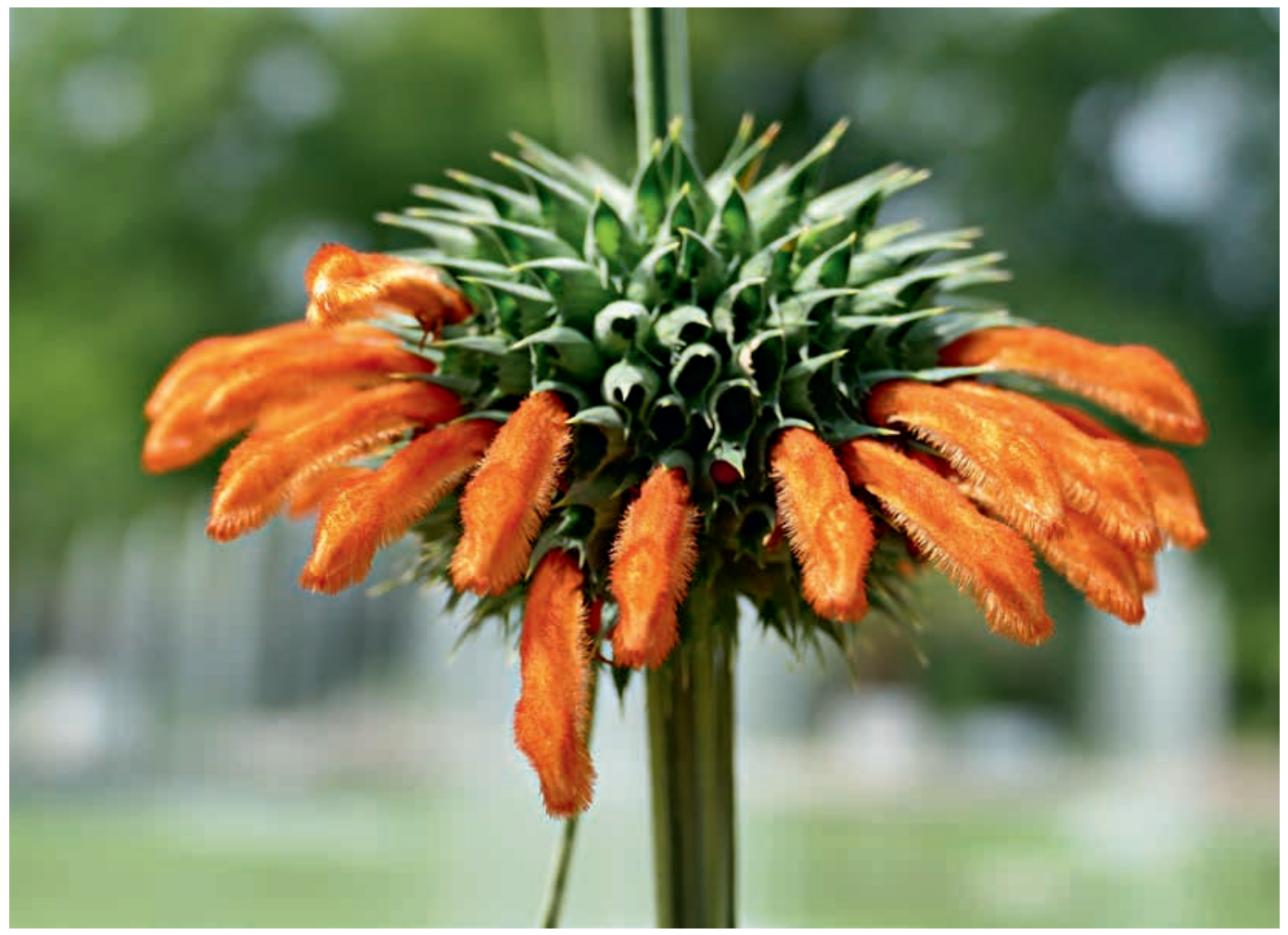

Abb. 9: Quirlförmiger Blütenstand von Leonotis nepetifolia. (Foto: H. STEInecke)

ist aber noch ziemlich unbekannt und erweckte bei vielen Gästen großes Interesse. Das Löwenohr (Leonotis nepetifolia), auch klip dagga genannt, ist ein Lippenblütler und damit auch mit Salbei verwandt. Die Heimat ist das tropische Afrika und südliche Indien, Leonotis nepetifolia wurde aber auch in andere tropische Gebiete eingeschleppt. Die Pflanze ist ein- bis mehrjährig, bei uns wird sie als einjährige Sommerblume gezogen. Sie erträgt Sonne und hohe Temperaturen sehr gut, weshalb sie sich bei den zu erwartenden häufiger auftretenden heißen Sommern als Sommerblume vermutlich immer mehr durchsetzen wird. Auffällig ist der vierkantige Stängel mit in Etagen angeordneten stechenden quirligen Blütenständen. Die schmalen, gebogenen Lippenblüten sind leuchtend orange gefärbt. In ihrer Heimat werden sie von Vögeln bestäubt.

Aufgrund ätherischer Öle duftet die Pflanze aromatisch. Sie enthält das Alkaloid Leonurin, das auf das zentrale Nervensystem eine beruhigende bis leicht euphorisierende Wirkung hat. In Afrika wird das Löwenohr volksmedizinisch verwendet, darunter wird es auch in Form von Tee verabreicht oder geraucht. In der traditionellen afrikanischen Medizin wird Leonotis außerdem gegen Schlangenbisse angewandt. Die ähnliche Art Leonotis leonurus aus dem südlichen Afrika ist ebenfalls eine für unsere Gärten geeignete Sommerblume.

\section{Anschrift der Autorin}

Dr. Hilke Steinecke, Palmengarten Frankfurt, Siesmayerstr. 61, 60323 Frankfurt, E-Mail: hilke.steinecke@stadt-frankfurt.de 